\title{
ЭКСПЕРИМЕНТАЛЬНЫЕ ИССЛЕДОВАНИЯ ДЕНДРИМЕРНОЙ НАНОКОНСТРУКЦИИ С RGD-ПЕПТИДОМ ДЛЯ РАДИОНУКЛИДНОЙ ТЕРАПИИ ОНКОЛОГИЧЕСКИХ ЗАБОЛЕВАНИЙ
}

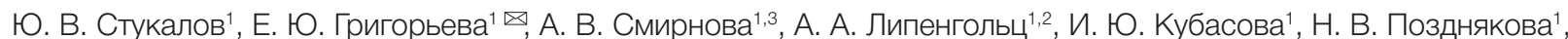
М. И. Лукашина ${ }^{4}$

${ }^{1}$ Национальный медицинский исследовательский центр онкологии имени Н. Н. Блохина, Москва

2 Федеральный медицинский биофизический центр имени А. И. Бурназяна, Москва

${ }^{3}$ Московский клинический научно-практический центр имени А. С. Логинова, Москва

${ }^{4}$ Национальный медицинский исследовательский центр детской гематологии, онкологии и иммунологии имени Дмитрия Рогачева, Москва

\begin{abstract}
Радионуклидная терапия (РНТ) является эффективным методом лечения множественных метастазов злокачественных опухолей. Расширение номенклатуры злокачественных новообразований, для которых возможно применение РНТ, происходит за счет создания новых туморотропных радиофармацевтических препаратов (РФП). Перспективно создание РФП на основе универсальной транспортной платформы, которая может быть модифицирована различными терапевтическими и диагностическими радионуклидами, а также векторными молекулами для достижения требуемой специфичности к опухолям и терапевтической эффективности. Целью работы было оценить в качестве такой транспортной платформы конструкцию на основе оригинального дендримера. Исследование на мышах показало его низкую токсичность (ЛД исследовали на примере наноконструкции (HK) из дендримера, RGD-пептида и радионуклида ${ }^{188} \mathrm{Re}\left({ }^{188} \mathrm{Re}-\mathrm{HK}\right)$. B качестве опухолевой модели использовали мышиную карциному легкого Льюиса LLC1. Данные биораспределения предложенной НК показали ее эффективное накопление в опухоли с коэффициентом диффееренциального накопления более 1 по отношению к основным органам и тканям и временем удержания в опухоли не менее 6 ч. Введение ${ }^{188} \mathrm{Re}$-HК в дозе 185 МБК/кг мышам с подкожно трансплантированной опухолью статистически достоверно $(p<0,05)$ способствовало торможению роста опухоли к 7-м суткам после введения до Т/C = 5\%, сохраняющемуся в течение 6 суток. Проведенные исследования показали перспективность исследованного дендримера как транспортной платформы для РНТ.
\end{abstract}

Ключевые слова: дендример, RGD-пептид, ${ }^{188} \mathrm{Re}$, радионуклидная терапия, биораспределение, исследование на животных, перевивные опухолевые модели

$\searrow$ Для корреспонденции: Елена Юрьевна Григорьева Каширское шоссе, д. 24, г. Москва, 115478; grig-elen11@mail.ru

Статья получена: 12.09.2018 Статья принята к печати: 11.10.2018

DOI: 10.24075 /vrgmu.2018.089

\section{EXPERIMENTAL STUDY OF DENDRIMER-BASED NANOPARTICLES WITH RGD-PEPTIDE FOR ANTICANCER RADIONUCLIDE THERAPY}

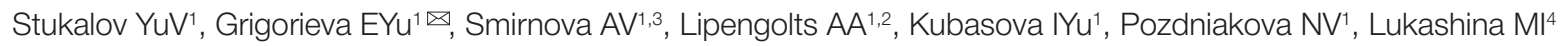 \\ 1 Blokhin National Medical Research Center of Oncology, Moscow \\ 2 Burnazyan Federal Medical Biophysical Center, Moscow \\ ${ }^{3}$ Loginov Moscow Clinical Scientific Center, Moscow \\ ${ }^{4}$ Dmitry Rogachev National Research Center of Pediatric Hematology, Oncology and Immunology, Moscow
}

Radionuclide therapy (RNT) is an effective modality for treating multiple metastases in patients with cancer. The list of malignancies that can be managed with RNT expands with the arrival of novel tumoritropic radiopharmaceuticals (RP). A versatile delivery platform capable of carrying various therapeutic and diagnostic radionuclides, as well as vector molecules needed to achieve sufficient specificity to tumor cells and ensure therapeutic efficacy may hold great promise for radiation therapy. The aim of this work was to assess the performance of a delivery system based on the original dendrimer. The dendrimer demonstrated low toxicity in mice $\left(\mathrm{LD}_{50}\right.$ was $\left.779 \pm 111 \mathrm{mg} / \mathrm{kg}\right)$. To study the specificity of the dendrimer to tumor cells and its therapeutic efficacy, we used a nanostructure (NS) composed of the dendrimer itself, the RGD peptide and ${ }^{188} \mathrm{Re}\left({ }^{188} \mathrm{Re}-\mathrm{NS}\right)$. Lewis lung carcinoma LLC1 was used as a tumor model. The biodistribution analysis revealed that the compound effectively accumulated in the tumor demonstrating a tumor-to-normal ratio $>1$ (relative to healthy organs and tissues) and retention time of at least 6 hours. Injections of $185 \mathrm{MBq} / \mathrm{kg}{ }^{188} \mathrm{Re}-\mathrm{NS}$ caused a statistically significant inhibition of tumor growth $(p<0.05)$ by day 7 following the injection ( $\mathrm{T} / \mathrm{C}=5 \%$ ), which remained stable for 6 days. Our findings suggest that the proposed dendrimer is a promising platform for RP delivery.

Keywords: dendrimer, RGD peptide, ${ }^{188} \mathrm{Re}$, radionuclide therapy, biodistribution, research in animals, transplanted tumor model

$\square$ Correspondence should be addressed: Elena Yu. Grigorieva

Kashirskoe shosse 24, Moscow, 115478; grig-elen11@mail.ru

Received: 12.09 .2018 Accepted: 11.10 .2018

DOI: 10.24075/brsmu.2018.089 
Наличие препаратов, способных эсфрективно и избирательно накапливаться в опухолевых тканях, является определяющим фактором в обеспечении терапевтической эффективности таких методов лечения злокачественных опухолей, как радионуклидная терапия, нейтрон-захватная и фотонзахватная виды терапии [1]. В последнее время большой интерес вызывает разработка препаратов на основе дендримеров [2-6] - шарообразных молекул 2-10 нм, содержащих во внешней сфере большое количество химических функциональных групп, позволяющих присоединять к дендримеру различные молекулы. На их основе можно создавать конструкции с присоединенным к дендримеру опухолеспецифичным агентом для обеспечения туморотропности и диагностическим и/или терапевтическим агентом для обеспечения функциональной пригодности. Ранее нами были показаны возможность и перспективность такого подхода для терапии и диагностики злокачественных опухолей [7-8]. Перспективным может быть подход с использованием рецепторов опухолевых клеток к некоторым низкомолекулярным соединениям. Так, конструкции на основе дендримеров с $\beta$-эстрадиолом показали эффективное накопление в трансплантированной аденокарциноме молочной железы Са755 [9]. В настоящей работе адресным компонентом конструкции служит RGD-пептид, который связывается с интегринами на поверхности опухолевых клеток [10-17].

Целью работы было экспериментально in vivo изучить возможности применения конструкций на основе созданных нами дендримеров в лучевой терапии и диагностике.

\section{МАТЕРИАЛЫ И МЕТОДЫ}

Исследованная наноконструкция (HK) представляет собой дендример первой генерации, к которому ковалентно присоединены сафранин для связывания перрената и RGD-пептид для обеспечения туморотропности. Дендример, лежащий в основе изучаемой НК, описан в более ранней работе [7]. На рис. 1 представлена описанная НK; ее строение подтверждено ПМР-спектроскопией.

В качестве терапевтического агента использовали радионуклид ${ }^{188} \mathrm{Re}$. Период полураспада ${ }^{188} \mathrm{Re}$ составляет 17 ч. Данный радионуклид при распаде испускает ß-излучение с энергией, равной 2,12 МэВ, обеспечивающее противоопухолевый эффект, и $\gamma$-излучение, в том числе с энергий 155 кэВ и выходом 15,2\%, позволяющим контролировать распределение РФП в организме больного с помощью $\gamma$-камеры [18-23]. Генераторная система ${ }^{188} \mathrm{~W} /{ }^{188} \mathrm{Re}$ позволяла продуцировать радионуклид ${ }^{188} \mathrm{Re}$ в течение 4-6 месяцев [24], поэтому проводить мечение соединений ${ }^{188} \mathrm{Re}$ возможно непосредственно перед применением РФП.

Мечение HK при помощи ${ }^{188} \mathrm{Re}$ осуществляли путем добавления перрената натрия, элюированного из генератора ${ }^{188} \mathrm{Re}$ ГРЕН-1 (АО ГНЦ РФ ФЭИ; Россия). При приготовлении рабочих растворов соединений количества ${ }^{188} \mathrm{RE}-\mathrm{HK}$ рассчитывали в соответствии с молярностью вносимого ${ }^{188} \mathrm{Re}\left(1 \mathrm{MБK}{ }^{188} \mathrm{Re}-0,00015\right.$ нM). Для полного связывания ${ }^{188}$ Re брали не менее чем 100-кратный избыток HK; радиоизотоп добавляли в исследуемые соединения ex tempore. ЯМР-спектры снимали на спектрометре WH-360 (Bruker; Германия) с рабочей частотой 360 МГц. Тонкослойную хроматограсию проводили с использованием пластинок с силикагелем типа Si 60 (размер частиц 5-17 мкм) (Fluka; США). Хроматограммы проявляли в парах йода.
Оценку острой токсичности ${ }^{188} \mathrm{RE}-\mathrm{HK}$ проводили только по токсичности исходного немодифицированного дендримера, вследствие высокой стоимости RGD-пептида. Токсикологические испытания дендримера проводили на здоровых самцах мышей линии Balb/c весом 19-21г (питомник ФГБУН «Научный центр биомедицинских технологий», филиал «Столбовая»; Россия). Все исследования на животных были выполнены в соответствии с этическими нормами обращения с животными [25].

Животных содержали при естественном освещении в стандартных условиях конвенционального вивария. Для проведения исследования формировали 10 групп одновозрастных животных по 6 особей в группе. Исследование проводили для 10 доз дендримера. Дендример, растворенный в 0,9\%-м растворе $\mathrm{NaCl}$ с 10\%-м содержанием ДМСО, вводили животным однократно интраперитонеально в объеме 0,2 мл раствора. Дозы дендримера на мышь составляли: 62,5 мг/кг; 125 мг/кг; 187,5 мг/Кг; $250 \mathrm{Mг/Кг;} 375 \mathrm{Mг/Кг;} 500$ мг/Кг; 750 мг/Кг; 1000 мг/Кг; 1250 мг/кг; 1500 мг/кг. Наблюдение за животными осуществляли в течение 30 дней, ежедневно отмечая изменения в их общем состоянии и поведении. Павших мышей вскрывали, оценивали степень изменения внутренних органов. По окончании эксперимента оставшихся в живых мышей подвергали эвтаназии методом цервикальной дислокации. Критериями оценки острой токсичности соединения служили: число павших животных и сроки их гибели, клиническая картина развивавшегося процесса и изменение поведенческих реакций, а также посмертные патологические изменения в тканях и внутренних органах (макроскопическая оценка) [26-28]. В дальнейшем, с использованием программы BioStat Pro 2008 5.0.1 (AnalystSoft; CША), были рассчитаны значения токсических доз.

Биораспределение созданной конструкции ${ }^{188} \mathrm{RE}-\mathrm{HK}$

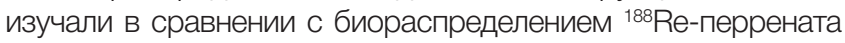

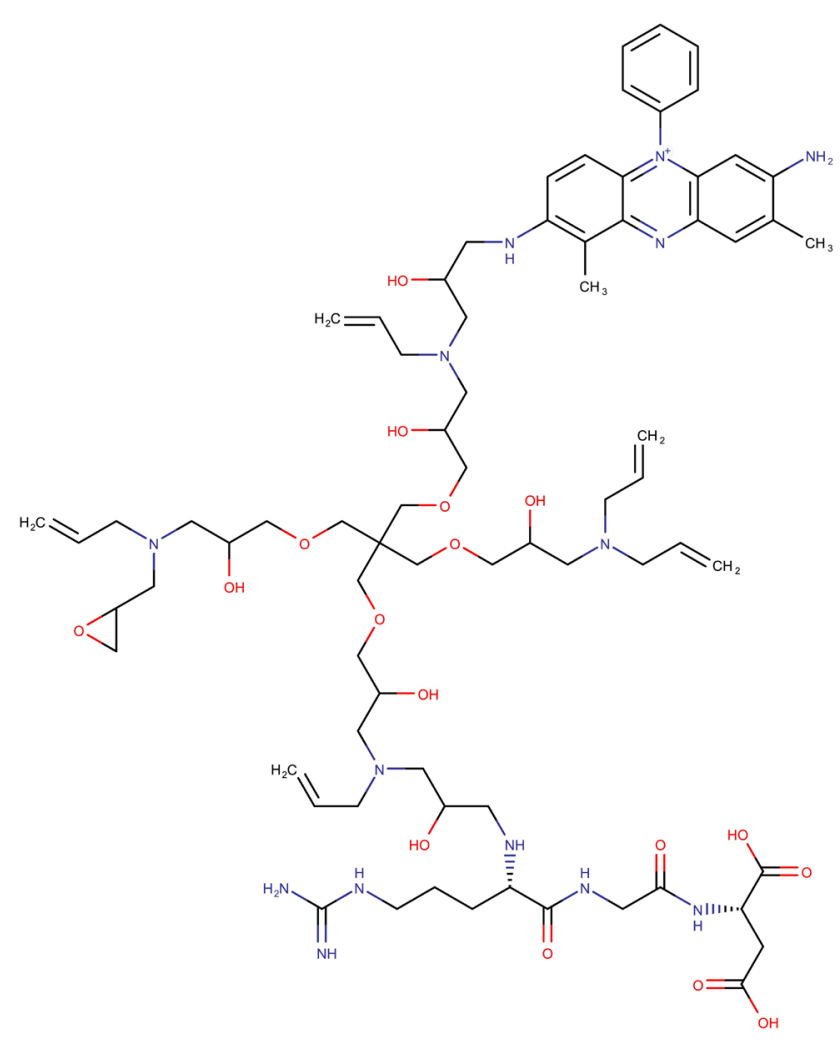

Рис. 1. Конструкция на основе дендримера с RGD-пептидом 
натрия на животных с подкожно (п/К) трансплантированной опухолью LLC1 (из коллекции опухолевых штаммов ФГБУ «НМИЦ онкологии им. Н. Н. Блохина»; Россия). Выбор данного штамма был обусловлен данными о равномерном распределении в опухоли рецептора $\alpha v \beta 3$, который является RGD-связывающим интегрином [29]. Мышей-самцов C57BI/6 весом 19-21 г группировали по 6 особей на каждую группу животных. Суспензию опухолевых клеток (4 000000 кл./ мышь) вводили под кожу правого бедра. На 10-й день после трансплантации LLC1 вводили внутривенно по 0,2 мл исследуемых соединений. Количество радиоактивности каждого вводимого соединения составляло 1,85 МБК на одно животное (92,5 МБК/кг). Для отбора образцов тканей животных декапитировали и проводили аутопсию с взятием артериальной крови. Аутопсию проводили через 1, 3, 6, 9, 12 и 24 ч после однократного введения. Дозы радиоактивности вводимых соединений измеряли на дозкалибраторе IZOMED 2010 (MED Nuklear-Medizintechnik Dresden $\mathrm{GmbH}$; Германия). Распределение исследовали с помощью прямой радиометрии биологических образцов. Уровень радиоактивности измеряли на сцинтилляционном автоматическом гамма-счетчике WIZARD 2480 (Perkin Elmer; США). Оценку результатов накопления осуществляли по содержанию ${ }^{188}$ Re в 1 г ткани/органа в процентах от введенного количества.

Изучение терапевтической эфффективности ${ }^{188} \mathrm{RE}-\mathrm{HK}$ проводили на мышах-самцах линии C57Bl/6 весом 20-22 г. Мышей группировали по 8 особей. Через двое суток после п/К трансплантации LLC1 животным одноразово системно

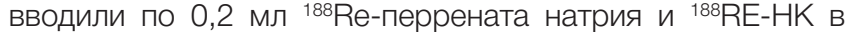
0,9\%-м растворе $\mathrm{NaCl}$. Были исследованы следующие дозы по активности ${ }^{188}$ Re: 15 МБК/кг, 92,5 МБК/кг, 185 МБК/Кг

Таблица 1. Распределение во времени ${ }^{188} \mathrm{Re}-\mathrm{HK}$ и ${ }^{188} \mathrm{Re}$ в органах и тканях мышей с подкожно трансплантированной LLC1 (в \% от введенного количества на 1 горгана/ткани)

\begin{tabular}{|c|c|c|c|c|c|c|c|}
\hline \multirow{2}{*}{ Наименование органа, ткани } & \multirow{2}{*}{ Соединение } & \multicolumn{6}{|c|}{ Время после введения соединения } \\
\hline & & 14 & 34 & 64 & 94 & 124 & 244 \\
\hline \multirow{3}{*}{ Кровь } & ${ }^{188} \mathrm{Re}-\mathrm{HK}$ & $14,90 \pm 0,20$ & $10,40 \pm 0,51$ & $7,76 \pm 0,25$ & $4,42 \pm 0,24$ & $2,31 \pm 0,20$ & $1,32 \pm 0,12$ \\
\hline & \multirow{2}{*}{${ }^{188} \mathrm{Re}$} & $15,04 \pm 0,12$ & $9,40 \pm 0,80$ & $7,36 \pm 0,20$ & $5,00 \pm 0,34$ & $2,64 \pm 0,48$ & $0,94 \pm 0,24$ \\
\hline & & $p>0,2$ & $p>0,2$ & $p>0,1$ & $p>0,1$ & $p>0,2$ & $p>0,1$ \\
\hline \multirow{3}{*}{ Печень } & ${ }^{188} \mathrm{Re}-\mathrm{HK}$ & $5,22 \pm 0,36$ & $4,09 \pm 0,13$ & $3,69 \pm 0,23$ & $3,06 \pm 0,25$ & $1,02 \pm 0,07$ & $0,07 \pm 0,01$ \\
\hline & \multirow{2}{*}{${ }^{188} \mathrm{Re}$} & $5,44 \pm 0,29$ & $4,66 \pm 0,42$ & $3,53 \pm 0,18$ & $1,98 \pm 0,51$ & $1,36 \pm 0,24$ & $0,15 \pm 0,05$ \\
\hline & & $p>0,2$ & $p>0,2$ & $p>0,2$ & $p>0,1$ & $p>0,1$ & $p>0,1$ \\
\hline \multirow{3}{*}{ Почки } & ${ }^{188} \mathrm{Re}-\mathrm{HK}$ & $7,27 \pm 0,42$ & $5,04 \pm 0,21$ & $4,47 \pm 0,29$ & $3,32 \pm 0,35$ & $1,84 \pm 0,19$ & $0,35 \pm 0,07$ \\
\hline & \multirow{2}{*}{${ }^{188} \mathrm{Re}$} & $7,34 \pm 0,46$ & $5,35 \pm 0,56$ & $3,67 \pm 0,35$ & $2,08 \pm 0,41$ & $1,44 \pm 0,25$ & $0,35 \pm 0,07$ \\
\hline & & $p>0,8$ & $p>0,5$ & $p>0,1$ & $p>0,1$ & $p>0,1$ & $p>0,6$ \\
\hline \multirow{3}{*}{ Легкие } & ${ }^{188} \mathrm{Re}-\mathrm{HK}$ & $10,01 \pm 0,50$ & $7,46 \pm 0,31$ & $5,68 \pm 0,16$ & $4,61 \pm 0,32$ & $2,75 \pm 0,23$ & $0,34 \pm 0,06$ \\
\hline & \multirow{2}{*}{${ }^{188} \mathrm{Re}$} & $10,09 \pm 0,50$ & $7,79 \pm 0,52$ & $5,52 \pm 0,26$ & $3,31 \pm 0,38$ & $1,99 \pm 0,33$ & $0,30 \pm 0,05$ \\
\hline & & $p>0,5$ & $p>0,2$ & $p>0,3$ & $p>0,1$ & $p>0,1$ & $p>0,2$ \\
\hline \multirow{3}{*}{ Селезенка } & ${ }^{188} \mathrm{Re}-\mathrm{HK}$ & $9,77 \pm 1,17$ & $7,66 \pm 0,43$ & $4,74 \pm 0,35$ & $4,15 \pm 0,30$ & $2,16 \pm 0,06$ & $0,29 \pm 0,05$ \\
\hline & \multirow{2}{*}{${ }^{188} \mathrm{Re}$} & $9,52 \pm 0,40$ & $6,90 \pm 0,37$ & $4,72 \pm 0,47$ & $3,01 \pm 0,20$ & $1,95 \pm 0,33$ & $0,31 \pm 0,11$ \\
\hline & & $p>0,8$ & $p>0,1$ & $p>0,8$ & $p>0,1$ & $p>0,2$ & $p>0,8$ \\
\hline \multirow{3}{*}{ Кость бедренная } & ${ }^{188} \mathrm{Re}-\mathrm{HK}$ & $5,61 \pm 5,61$ & $3,77 \pm 3,77$ & $3,14 \pm 3,14$ & $2,13 \pm 2,13$ & $1,45 \pm 1,45$ & $0,44 \pm 0,44$ \\
\hline & \multirow{2}{*}{${ }^{188} \mathrm{Re}$} & $5,02 \pm 0,29$ & $3,98 \pm 0,38$ & $2,77 \pm 0,21$ & $1,58 \pm 0,06$ & $1,11 \pm 0,09$ & $0,44 \pm 0,05$ \\
\hline & & $p>0,1$ & $p>0,5$ & $p>0,1$ & $p>0,1$ & $p>0,1$ & $p>0,8$ \\
\hline \multirow{3}{*}{ Опухоль } & ${ }^{188} \mathrm{Re}-\mathrm{HK}$ & $8,16 \pm 0,26$ & $8,20 \pm 0,14$ & $8,24 \pm 0,06$ & $6,54 \pm 0,35$ & $3,15 \pm 0,35$ & $1,17 \pm 0,07$ \\
\hline & \multirow{2}{*}{${ }^{188} \mathrm{Re}$} & $6,06 \pm 0,17$ & $3,82 \pm 0,26$ & $2,32 \pm 0,42$ & $1,90 \pm 0,16$ & $1,14 \pm 0,09$ & $0,66 \pm 0,07$ \\
\hline & & $p<0,03$ & $p<0,01$ & $p<0,01$ & $p<0,01$ & $p<0,05$ & $p<0,05$ \\
\hline
\end{tabular}

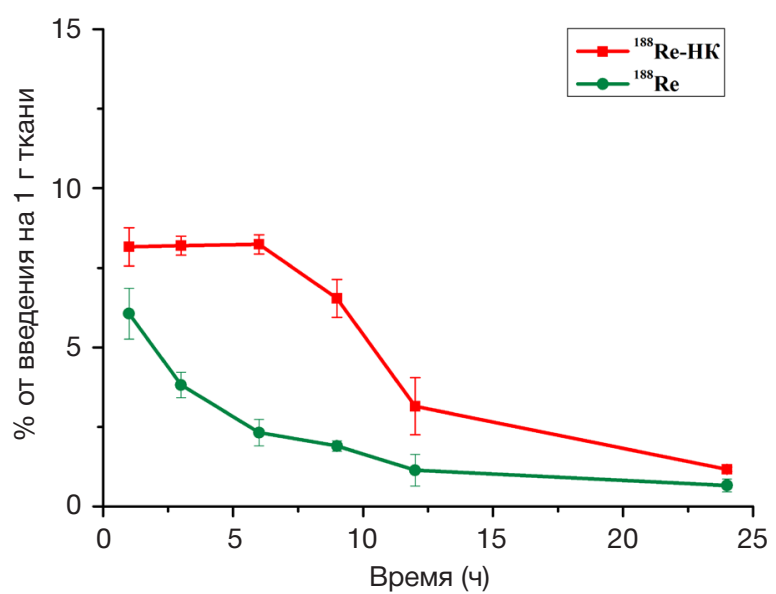

Рис. 2. Динамика накопления (\% от введенной активности на 1 г ткани) ${ }^{188}$ Re-HK и ${ }^{188}$ Re-перрената натрия у мышей-самцов C57BI/6 в подкожно трансплантированной LLC1 
(0,3 МБк, 1,85 МБк, 3,7 МБк на животное). В контрольной группе вводили 0,2 мл 0,9\%-го $\mathrm{NaCl}$. В течение всего эксперимента ежедневно измеряли размеры опухолей и их объемы. По полученным данным в группах контроля и эксперимента рассчитывали стандартный количественный показатель противоопухолевого действия T/C по формуле [30]:

$$
\mathrm{T} / \mathrm{C}=100 \cdot V_{\text {опьт }} / V_{\text {контр }}
$$

где $V_{\text {опыт }}$ - средний объем опухолей у животных в экспериментальной группе, $V_{\text {контр }}-$ средний объем опухолей у животных в контрольной группе.

Для анализа данных использовали программы OriginPro 8.0 (OriginLab; США) и Excel 2003 (Microsoft; CШA). Статистическую значимость полученных результатов определяли при помощи непараметрического U-критерия Манна-Уитни, а различия считали статистически значимыми при $p \leq 0,05$.

\section{РЕЗУЛЬТАТЫ ИССЛЕДОВАНИЯ}

Критериями оценки наличия острой токсичности соединения служили число павших животных и сроки их гибели с момента введения дендримера. Было установлено, что гибель животных начинается при введении дозы дендримера 500 мг/кг (2 из 6 животных), 4 из 6 животных погибают при дозе 1000 мг/кг и все животные в группе погибают при дозе 1500 мг/кг. При введении летальных доз дендримера мышам в течение первых 30 мин наблюдали транзиторное двигательное возбуждение, переходящее в сопорозное состояние. Смерть животных наступала в зависимости от дозы дендримера в течение 144 ч. У выживших мышей в течение первых 5 дней после введения соединения отмечали транзиторную потерю массы тела (5-8\%) и проявление повышенной двигательной активности. При проведении некропсии животных было отмечено следующее: сердце, почки, селезенка и легкие - без визуально выраженной патологии; брюшина - без видимых изменений и гиперемии; печень - увеличена в размерах, края притуплены. При введении в сублетальных дозах соединение не вызывало видимых нарушений состояния и поведения животных: признаков атаксии или местных парезов не наблюдали. Мыши прибавляли в весе в соответствии с динамикой контрольной группы, состояние видимых кожных покровов не изменялось. При аутопсии выведенных из эксперимента животных видимых изменений внутренних органов не выявили. На основании данных о количестве павших животных в каждой из 10 экспериментальных групп были рассчитаны токсические дозы дендримера для мышей: ЛД $10270 \pm 92$ мг/кг; ЛД $=382 \pm 94$ мг/Кг; ЛД $=779 \pm 111 \mathrm{мг/Кг;} \mathrm{ЛД}$ 196 мг/Кг; ЛД ${ }_{90}=1289 \pm 260$ мг/кг; ЛД $100=1376 \pm 367$ мг/Кг.

Результаты распределения ${ }^{188} \mathrm{RE}-\mathrm{HK}$ в сравнении

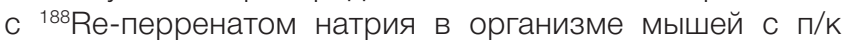
трансплантированной LLC1 представлены в табл. 1. Статистически значимые различия были выявлены только при анализе данных о накоплении соединений в опухоли. Динамика накопления ${ }^{188} \mathrm{Re}-\mathrm{HK}$ и ${ }^{188} \mathrm{Re}$-перрената натрия в образцах LLC1 представлена на рис. 2.

В связи с тем что ${ }^{188} \mathrm{Re}-\mathrm{HK}$ разрабатывается для противоопухолевой радиотерапии, важной фармакокинетической характеристикой является дифференциальный уровень накопления данного соединения в опухоли. С этой целью были рассчитаны величины коэффициентов дифференциального накопления (КДН) - отношения удельной активности опухоли к удельной активности других органов и тканей (табл. 2). Почти для всех органов и тканей после 3 ч величина отношений для ${ }^{188} \mathrm{Re}-\mathrm{HK}$ выше 1 , что свидетельствует о более интенсивном выведении соединения из органов по сравнению с опухолью. В то же время для ${ }^{188} \mathrm{Re}$ скорость выведения из опухоли была сопоставима с выведением из печени и костной ткани.

Терапевтическую эффективность определяли в течение месяца после трансплантации опухоли животным.

Таблица 2. Сравнительные данные дифференциального уровня накопления ${ }^{188} \mathrm{Re}-\mathrm{HK}$ и ${ }^{188} \mathrm{Re}$ в органах и тканях мышей

\begin{tabular}{|c|c|c|c|c|c|c|c|}
\hline \multirow{2}{*}{ Наименование органа, ткани } & \multirow{2}{*}{ Соединение } & \multicolumn{6}{|c|}{ Время после введения соединения } \\
\hline & & 14 & 34 & 64 & 94 & 124 & 244 \\
\hline \multirow{3}{*}{ Опухоль/кровь } & ${ }^{188} \mathrm{Re}-\mathrm{HK}$ & $0,55 \pm 0,02$ & $0,79 \pm 0,04$ & $1,06 \pm 0,04$ & $1,48 \pm 0,04$ & $1,36 \pm 0,06$ & $0,89 \pm 0,11$ \\
\hline & \multirow{2}{*}{${ }^{188} \mathrm{Re}$} & $0,40 \pm 0,01$ & $0,41 \pm 0,06$ & $0,32 \pm 0,07$ & $0,38 \pm 0,05$ & $0,45 \pm 0,11$ & $0,72 \pm 0,18$ \\
\hline & & $p>0,1$ & $p<0,05$ & $p<0,004$ & $p<0,004$ & $p<0,004$ & $p>0,1$ \\
\hline \multirow{3}{*}{ Опухоль/печень } & ${ }^{188} \mathrm{Re}-\mathrm{HK}$ & $1,57 \pm 0,14$ & $2,01 \pm 0,09$ & $2,24 \pm 0,13$ & $2,15 \pm 0,24$ & $3,09 \pm 0,29$ & $16,55 \pm 2,7$ \\
\hline & \multirow{2}{*}{${ }^{188} \mathrm{Re}$} & $1,11 \pm 0,04$ & $0,82 \pm 0,02$ & $0,66 \pm 0,14$ & $1,02 \pm 0,37$ & $0,86 \pm 0,19$ & $4,65 \pm 1,25$ \\
\hline & & $p<0,05$ & $p<0,004$ & $p<0,001$ & $p<0,004$ & $p<0,001$ & $p<0,001$ \\
\hline \multirow{3}{*}{ Опухоль/почки } & ${ }^{188} \mathrm{Re}-\mathrm{HK}$ & $1,12 \pm 0,07$ & $1,63 \pm 0,04$ & $1,85 \pm 0,11$ & $1,99 \pm 0,22$ & $1,73 \pm 0,30$ & $3,42 \pm 0,85$ \\
\hline & \multirow{2}{*}{${ }^{188} \mathrm{Re}$} & $0,83 \pm 0,07$ & $0,72 \pm 0,08$ & $0,63 \pm 0,09$ & $0,95 \pm 0,27$ & $0,81 \pm 0,19$ & $1,91 \pm 0,15$ \\
\hline & & $p<0,004$ & $p<0,004$ & $p<0,004$ & $p<0,004$ & $p<0,05$ & $p<0,05$ \\
\hline \multirow{3}{*}{ Опухоль/легкие } & ${ }^{188} \mathrm{Re}-\mathrm{HK}$ & $0,82 \pm 0,06$ & $1,10 \pm 0,06$ & $1,45 \pm 0,05$ & $1,43 \pm 0,17$ & $3,86 \pm 0,80$ & $13,92 \pm 2,56$ \\
\hline & \multirow{2}{*}{${ }^{188} \mathrm{Re}$} & $0,60 \pm 0,04$ & $0,49 \pm 0,05$ & $0,42 \pm 0,09$ & $0,58 \pm 0,10$ & $0,59 \pm 0,14$ & $2,18 \pm 0,17$ \\
\hline & & $p<0,05$ & $p<0,004$ & $p<0,004$ & $p<0,004$ & $p<0,02$ & $p<0,002$ \\
\hline \multirow{3}{*}{ Опухоль/селезенка } & ${ }^{188} \mathrm{Re}-\mathrm{HK}$ & $0,84 \pm 0,11$ & $1,07 \pm 0,04$ & $1,75 \pm 0,12$ & $1,58 \pm 0,05$ & $1,46 \pm 0,12$ & $4,09 \pm 0,80$ \\
\hline & \multirow{2}{*}{${ }^{188} \mathrm{Re}$} & $0,64 \pm 0,04$ & $0,55 \pm 0,02$ & $0,49 \pm 0,04$ & $0,63 \pm 0,08$ & $0,60 \pm 0,14$ & $2,26 \pm 0,64$ \\
\hline & & $p<0,05$ & $p<0,004$ & $p<0,004$ & $p<0,004$ & $p<0,004$ & $p<0,004$ \\
\hline \multirow{3}{*}{ Опухоль/кость бедренная } & ${ }^{188} \mathrm{Re}-\mathrm{HK}$ & $1,46 \pm 0,10$ & $2,17 \pm 0,01$ & $2,64 \pm 0,23$ & $3,08 \pm 0,22$ & $2,18 \pm 0,32$ & $2,67 \pm 0,15$ \\
\hline & \multirow{2}{*}{${ }^{188} \mathrm{Re}$} & $1,21 \pm 0,10$ & $0,97 \pm 0,13$ & $0,85 \pm 0,21$ & $1,20 \pm 0,14$ & $1,04 \pm 0,14$ & $1,51 \pm 0,31$ \\
\hline & & $p<0,05$ & $p<0,004$ & $p<0,004$ & $p<0,004$ & $p<0,05$ & $p<0,004$ \\
\hline \multicolumn{2}{|l|}{ Опухоль 188} & $1,35 \pm 0,05$ & $2,15 \pm 0,19$ & $3,55 \pm 0,66$ & $3,45 \pm 0,32$ & $2,76 \pm 0,51$ & $1,79 \pm 0,24$ \\
\hline
\end{tabular}


При однократном введении ${ }^{188} \mathrm{Re}-\mathrm{HK}$ выраженный противоопухолевый эффект $(p<0,05)$ на всех сроках наблюдения имел место только при введении дозы 185 МБК/кг (табл. 3, рис. ЗА). После введения животным ${ }^{188} R$ еперрената натрия в дозе 185 МБК/Кг терапевтического эффекта не наблюдали (рис. ЗБ). Значения количественного параметра противоопухолевого эффекта Т/C для исследованных доз 188Rе представлены в табл. 3.

Наблюдаемый противоопухолевый эффект носил дозозависимый характер: в использованном диапазоне доз ${ }^{188} \mathrm{Re}-\mathrm{HK}$ при максимальной дозе, равной 185 МБК/ кГ, наблюдали значимый противоопухолевый эффект; при промежуточной дозе, равной 92,5 МБК/Кг, слабовыраженный и при дозе 15 МБК/кг - незначительный. Представленные кривые роста опухолей наглядно иллюстрируют динамику описанных процессов. Таким образом, были определены минимально эффективные концентрации ${ }^{188} \mathrm{Re}-\mathrm{HK}$ для достижения терапевтического эффекта.

\section{ОБСУЖДЕНИЕ РЕЗУЛЬТАТОВ}

В результате проведенных исследований было установлено, что токсичность предложенного дендримера сопоставима с токсичностью аналогичных дендримеров данного класса, исследованных ранее [31].

Полученные нами результаты свидетельствуют о том, что ${ }^{188} \mathrm{Re}-\mathrm{HK}$, попадая в опухоль, удерживается тканями опухоли длительное время: до 6 ч после введения соединения. На протяжении 6 ч содержание ${ }^{188} \mathrm{Re}-\mathrm{HK}$ в опухоли остается достаточно высоким и составляет 8,2\% от введенного количества. Это может свидетельствовать о прочном связывании ${ }^{188} \mathrm{Re}-\mathrm{HK}$ со структурами опухолевой ткани. При аналогичном анализе динамики накопления ${ }^{188} \mathrm{Re}$-перрената натрия такого эффекта не наблюдалось. Максимальное соотношение активностей ${ }^{188} \mathrm{Re}-\mathrm{HK}$ и ${ }^{188} \mathrm{Re}$-перрената натрия в опухолевых тканях составило 3,55 \pm 0,660 через 6 ч после введения.
Полученный in vivo значимый противоопухолевый эффрект при дозе ${ }^{188} \mathrm{Re}-\mathrm{HK}$, равной 185 МБК/кг, с учетом коэффициентов межвидового пересчета составляет для человека 15,42 МБК/кг, тогда как типичные значения терапевтических доз существующих РФП достигают 44-47 МБК/кг [32]. Это позволяет снизить лучевую нагрузку на пациента при сохранении терапевтической эффективности. Еще одно преимущество предлагаемой нами конструкции - ее низкая токсичность. В нашем исследовании для полного связывания ${ }^{188} R e$ использовали 100-кратный избыток НК; доза НК при 185 МБК/кг составляет 4,05 • $10^{-3}$ мг/Кг (или 2,16 • 10-3 мг/Кг по дендримеру), что ниже ЛД10 на 5 порядков. В совокупности описанные эфффекты позволяют рассматривать предложенную НК как перспективную для создания РФП.

\section{ВЫВОДЫ}

В результате проведенного исследования была показана принципиальная возможность применения конструкций на основе созданных дендримеров как средства направленной доставки противоопухолевого агента, определен минимальный порог терапевтической дозы ${ }^{188} \mathrm{Re}$ в полученном соединении и показано, что действие синтезированного дендримера носит дозозависимый характер. Исследование острой токсичности на мышах показало низкую токсичность конструкции ${ }^{188} \mathrm{Re}-\mathrm{HK}$. Установлена низкая токсичность исследуемого соединения, позволяющая достигать значимого противоопухолевого эффекта при вводимых дозах намного меньше дозы ЛД10. Выявленные уровни накопления и время удержания ${ }^{188} \mathrm{Re}-\mathrm{HK}$ в опухолевой ткани дают основание предполагать возможность достижения высокой терапевтической дозы $\beta$-излучения в патологическом очаге при ее использовании. Введение ${ }^{188} \mathrm{Re}$ в составе ${ }^{188} \mathrm{Re}-\mathrm{HK}$ в дозе 185 МБК/кг вызывает значимый противоопухолевый эффект в течение 18 дней после лечения.

Таблица 3. Оценка эффективности противоопухолевого действия ${ }^{188} \mathrm{Re}-\mathrm{HK}$ на подкожно трансплантированную опухоль LLC1

\begin{tabular}{|l|c|c|c|c|c|c|c|c|c|}
\hline Время после введения, сут. & \multicolumn{3}{|c|}{7 сут. } & \multicolumn{3}{|c|}{14 сут. } & \multicolumn{3}{c|}{18 сут. } \\
\hline Доза активности по ${ }^{188} \mathrm{Re}$, МБк/кг & 15 & 92,5 & 185 & 15 & 92,5 & 185 & 15 & 92,5 & 185 \\
\hline T/C, \% & 100 & 41 & 5 & 74 & 55 & 13 & 87 & 75 & 20 \\
\hline
\end{tabular}

Примечание. Сроки оценки показателей эффективности даны на 7-е, 14-е и 18-е сутки после лечения.

A

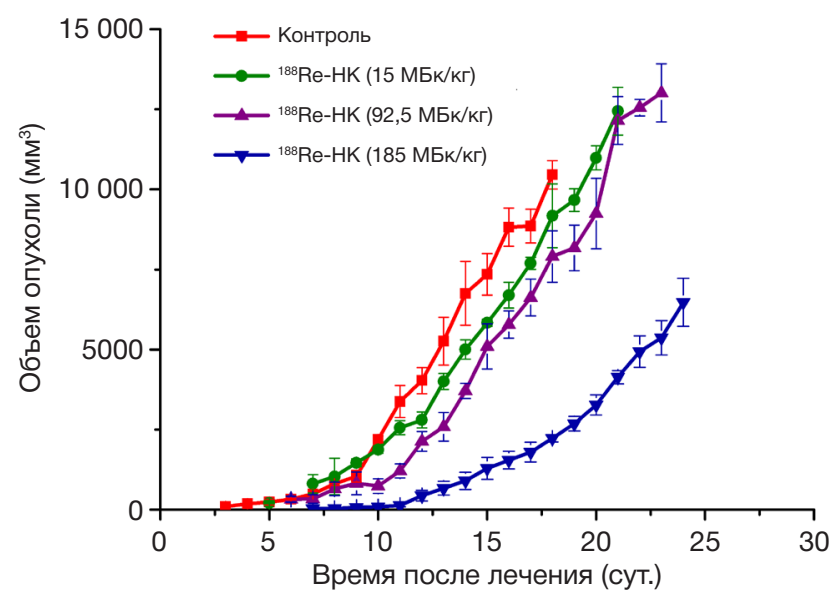

Б

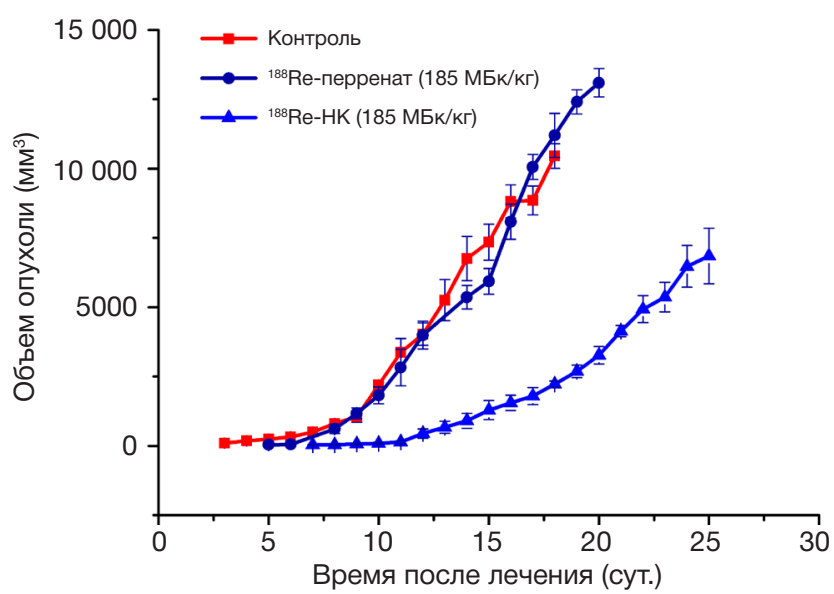

Рис. 3. Динамика роста п/K трансплантированной опухоли LLC1 в организме мышей C57BI/6 после однократного введения ${ }^{188}$ Re-HK с различными дозами ${ }^{188} \operatorname{Re}(\mathrm{A}) ;$ после однократного введения 188Re-перрената натрия и ${ }^{188} \mathrm{Re}-\mathrm{HK}$ (доза ${ }^{188} \mathrm{Re}=185 \mathrm{MБK} / \mathrm{K}$ ) (Б) 


\section{Литература}

1. Kulakov VN, Lipengolts AA, Grigorieva EY, Shimanovskii NL. Pharmaceuticals for Binary Radiotherapy and Their Use for Treatment of Malignancies (A Review). Pharm Chem J. 2016 Sep 8; 50 (6): 388-93.

2. Rakesh Kumar Tekade, Palanirajan Vijayaraj Kumar, Narendra Kumar Jain. Dendrimers in Oncology: An Expanding Horizon. Chem Rev. 2009; 109: 49-87.

3. Wu L-P, Ficker M, Christensen JB. Dendrimers in Medicine: Therapeutic Concepts and Pharmaceutical Challenges. Bioconjugate Chem. 2015; 26: 1198-211.

4. Olson ES, Jiang T, Aguilera T, Nguyen QT, Ellies LG, Scadeng M, et al. Activatable cell penetrating peptides linked to nanoparticles as dual probes for in vivo fluorescence and MR imaging of proteases. Proc Natl Acad Sci. 2010; 107 (9): 4311-6.

5. Palmerston Mendes L, Pan J, Torchilin V. Dendrimers as Nanocarriers for Nucleic Acid and Drug Delivery in Cancer Therapy. Molecules. 2017; 22 (9): 1401.

6. $\mathrm{Xu} L$, Yeudall WA, Yang $\mathrm{H}$. Folic acid-decorated polyamidoamine dendrimer exhibits high tumor uptake and sustained highly localized retention in solid tumors: Its utility for local siRNA delivery. Acta Biomater. 2017; (57): 251-61.

7. Stukalov YuV, Grigorieva EYu, Kulakov VN, Baryshnikov AYu. Transport platform synthesis for drug development. Pharmaceutical chemistry journal. 2017; 51 (6): 480-1.

8. Григорьева Е. Ю., Стукалов Ю. В., Смирнова А. В., Колдаева Е. Ю., Калыгина Н. С., Кулаков В. Н. и др. Цитотоксическое действие конструкций на основе дендримеров с 188Re c моноклональными антителами антиICO-25 (MUC1) и анти-ICO-80 (CD5) На моделях клеточных линий SKOV-3 (рак яичника) и Jurkat (Т-лимфобластная лимфома). Химико-фармацевтический журнал. 2018; 52 (8): 3-6.

9. Григорьева Е. Ю., Стукалов Ю. В., Колдаева Е. Ю. Конструкции на основе дендримеров нового класса для таргетной радиотерапии онкологических заболеваний. Российский биотерапевтический журнал. 2009; 8 (1): 5-6.

10. Domingo-Espín J, Petegnief V, de Vera N. RGD-based cell ligands for cell-targeted drug delivery act as potent trophic factors. Nanomedicine: Nanotechnology, Biology, and Medicine. 2012; (8): 1263-6.

11. Берман А. Е., Козлова Н. И., Морозевич Г. Е. Интегринь как потенциальная мишень для целевой терапии рака. Биомедицинская химия. 2013; 59 (3): 239-48.

12. Miller LM, Pritchard JM, Macdonald SJF, Jamieson C, Watson AJB. The Emergence of Small Molecule Non-RGD-mimetic Inhibitors for RGD Integrins Journal of Medicinal Chemistry. 2017; 60 (8): 3241-51.

13. Hwang R, Varner J. The role of integrins in tumor angiogenesis. Hematol Oncol Clin North Am. 2004; 18 (5): 991-1006.

14. Koistinen $P$, Ahonen $M$, Kähäri VM, Heino J. AlphaV integrin promotes in vitro and in vivo survival of cells in metastatic melanoma. Int J Cancer. 2004; 112 (1): 61-70.

15. Kuphal S, Bauer R, Bosserhoff AK. Integrin signaling in malignant melanoma. Cancer Metastasis Rev. 2005; 24 (2): 195-222.

16. Veeravagu A, Liu Z, Niu G, Chen K, Jia B, Cai W et al. Integrin alphavbeta3-targeted radioimmunotherapy of glioblastoma multiforme. Clin Cancer Res. 2008; (14): 7330-9.

17. Landen CN, Kim TJ, Lin YG, Merritt WM, Kamat AA, Han LY et al. Tumour-selective response to antibody-mediated targeting of alphavbeta3 integrin in ovarian cancer. Neoplasia. 2008; 10 (11): 1259-67

18. Beiki D, Tajik M, Haddad P, Fallahi B, Arefpour AM, Mirzaei H et. al. Effectiveness and complications of 188Re-HEDP in palliative treatment of diffuse skeletal metastases. Iran J Nucl Med. 2015; 23 (1): 44-8.

19. Erfani M, Doroudi A, Dinari MA, Shirmardi SP. Preparation of a rhenium-188 labeled bisphosphonate for bone pain palliation therapy. Journal of Radioanalytical and Nuclear Chemistry. 2015; 303 (3): 2027-32

20. Zamora PO, Gulhke S, Bender H. Experimental radiotherapy of receptor-positive human prostate adenocarcinoma with $188 \mathrm{Re}$ RC-160, a directly-radiolabeled somatostatin analogue. Int $J$ Cancer. 1999; (6): 214-20.

21. Molina-Trinidad EM, Arteaga de Murphy C, Ferro-Flores G. Radiopharmacokinetic and dosimetric parameters of $188 \mathrm{Re}$ lanreotide in athymic mice with induced human cancer tumors. Int J Pharm. 2006; (310): 125-30.

22. Krylov V, Kochetova T, Smolyarchuk M, Petriev V, Kanygin V, Alexandrova $\mathrm{A}$ et al. Evaluation of 188Re-khedp (phosphoren) for palliative treatment in patients with bone metastases., World Journal of Nuclear Medicine. 2013; 12 (2): 255-6.

23. Kochetova T, Smolyarchuk M, Krylov V, Voloznev V, Lunev A, Petriev VM. 188Re-zoledronic acid: first in man study. World Journal of Nuclear Medicine. 2013; 12 (2): 228-9.

24. Konior M, Iller E. Classic Radionuclide 188W/188Re Generator (Experiments, Design and Construction). Mod Chem appl. 2014; (2): 143.

25. Европейская конвенция по защите позвоночных животных, используемых для экспериментальных и других научных целей, ЕЭС, Страсбург, 1985 г. Ланималогия. 1993; (1): 29 с.

26. Беленький М. Л. Элементы количественной оценки фармакологического эффекта: Л.: Изд-во «МедГиз», 1968; 151 с.

27. Гуськова Т. А. Токсикология лекарственных средств: М.: Издво «Русский врач», 2003; 154 с

28. Трахтенберг И. А., Сова А. Е., Шефтель В. О., Оникиенко Ф. А. Проблема нормы в токсикологии. М.: Изд-во «Медицина», 1992; 208 c.

29. Seguin J, Nicolazzi C, Mignet N, Scherman D, Chabot GG. Vascular density and endothelial cell expression of integrin alpha $\checkmark$ beta 3 and E-selectin in murine tumours. Tumour Biol. 2012; 33 (5): 1709-17.

30. Трещалина Е. М., Жукова О. С., Герасимова Г. К., Андронова Н. В. и др. Методические рекомендации по доклиническому изучению противоопухолевой активности лекарственных средств. В книге: Миронов А. Н., Бунатян Н. Д. и др., редакторы. Руководство по проведению доклинических исследований лекарственных средств. М.: Гриф и К., 2012; с. 642-657.

31. Колдаева Е. Ю., Григорьева Е. Ю., Стукалов Ю. В. Сравнительная токсикология наноконструкций на основе дендримеров для таргетной радиотерапии в онкологии. Российский биотерапевтический журнал. 2010; 9 (3): 13-14.

32. Argyrou M, Valassi A, Andreou M, Lyra M. Rhenium-188 Production in Hospitals, by W-188/Re-188 Generator, for Easy Use in Radionuclide Therapy. Int J Mol Imaging. 2013; (2013): 1-7.

\section{References}

1. Kulakov VN, Lipengolts AA, Grigorieva EYu, Shimanovskii NL. Pharmaceuticals for Binary Radiotherapy and Their Use for Treatment of Malignancies (A Review). Pharm Chem J. 2016 Sep 8; 50 (6): 388-93.

2. Rakesh Kumar Tekade, Palanirajan Vijayaraj Kumar, Narendra Kumar Jain. Dendrimers in Oncology: An Expanding Horizon. Chem Rev. 2009; 109: 49-87.

3. Wu L-R, Ficker M, Christensen JB. Dendrimers in Medicine: Therapeutic Concepts and Pharmaceutical Challenges.
Bioconjugate Chem. 2015; 26: 1198-211.

4. Olson ES, Jiang T, Aguilera T, Nguyen QT, Ellies LG, Scadeng M, et al. Activatable cell penetrating peptides linked to nanoparticles as dual probes for in vivo fluorescence and MR imaging of proteases. Proc Natl Acad Sci. 2010; 107 (9): 4311-6.

5. Palmerston Mendes L, Pan J, Torchilin V. Dendrimers as Nanocarriers for Nucleic Acid and Drug Delivery in Cancer Therapy. Molecules. 2017; 22 (9): 1401.

6. $\mathrm{Xu} \mathrm{L,} \mathrm{Yeudall} \mathrm{WA,} \mathrm{Yang} \mathrm{H.} \mathrm{Folic} \mathrm{acid-decorated} \mathrm{polyamidoamine}$ 
dendrimer exhibits high tumor uptake and sustained highly localized retention in solid tumors: Its utility for local siRNA delivery. Acta Biomater. 2017; (57): 251-61.

7. Stukalov YuV, Grigorieva EYu, Kulakov VN, Baryshnikov AYu. Transport platform synthesis for drug development. Pharmaceutical chemistry journal. 2017; 51 (6): 480-1.

8. Grigorieva EYu, Stukalov YV, Smirnova AV, Koldaeva EY Kalygina NS, Kulakov VN et al. Cytotoxicity of Conjugates of 188Re-Labeled Dendrimers and Monoclonal Antibodies AntiICO-25 (MUC1) and Anti-ICO-80 (CD5) Against SKOV-3 (Ovary Cancer) and Jurkat Tumor Cell Lines (T-Lymphoblstic Lymphoma). Pharm Chem J. 2018; 52 (8): 681-4.

9. Grigorieva EYu, Stukalov YuV, Koldaeva EYu. Konstruktsii na osnove dendrimerov novogo klassa dlya targetnoy radioterapil onkologicheskikh zabolevaniy. Rossiyskiy bioterapevticheskiy zhurnal. 2009; 8 (1): 5-6. Russian.

10. Domingo-Espín J, Petegnief V, de Vera N. RGD-based cell ligands for cell-targeted drug delivery act as potent trophic factors. Nanomedicine: Nanotechnology, Biology, and Medicine. 2012; (8): 1263-6.

11. Berman AE, Kozlova, NI, Morozevich GE. Integrins as a potential target for targeted anticancer therapy. Biochem. Moscow Supp Ser B. 2012; 6 (3): 205-10.

12. Miller LM, Pritchard JM, Macdonald SJF, Jamieson C, Watson AJB The Emergence of Small Molecule Non-RGD-mimetic Inhibitors for RGD Integrins Journal of Medicinal Chemistry. 2017; 60 (8): 3241-51.

13. Hwang R, Varner $\mathrm{J}$. The role of integrins in tumor angiogenesis. Hematol Oncol Clin North Am. 2004; 18 (5): 991-1006.

14. Koistinen $\mathrm{P}$, Ahonen $\mathrm{M}$, Kähäri VM, Heino J. AlphaV integrin promotes in vitro and in vivo survival of cells in metastatic melanoma. Int J Cancer. 2004; 112 (1): 61-70.

15. Kuphal $\mathrm{S}$, Bauer $\mathrm{R}$, Bosserhoff AK. Integrin signaling in malignant melanoma. Cancer Metastasis Rev. 2005; 24 (2): 195-222.

16. Veeravagu A, Liu Z, Niu G, Chen K, Jia B, Cai W et al. Integrin alphavbeta3-targeted radioimmunotherapy of glioblastoma multiforme. Clin Cancer Res. 2008; (14): 7330-9.

17. Landen CN, Kim TJ, Lin YG, Merritt WM, Kamat AA, Han LY et al. Tumour-selective response to antibody-mediated targeting of alphavbeta3 integrin in ovarian cancer. Neoplasia. 2008; 10 (11): 1259-67.

18. Beiki D, Tajik M, Haddad P, Fallahi B, Arefpour AM, Mirzaei H et. al. Effectiveness and complications of 188Re-HEDP in palliative treatment of diffuse skeletal metastases. Iran J Nucl Med. 2015; $23(1): 44-8$.

19. Erfani M, Doroudi A, Dinari MA, Shirmardi SP. Preparation of a rhenium-188 labeled bisphosphonate for bone pain palliation therapy. Journal of Radioanalytical and Nuclear Chemistry. 2015; 303 (3): 2027-32

20. Zamora PO, Gulhke S, Bender H. Experimental radiotherapy of receptor-positive human prostate adenocarcinoma with 188ReRC-160, a directly-radiolabeled somatostatin analogue. Int J Cancer. 1999; (6): 214-20.

21. Molina-Trinidad EM, Arteaga de Murphy C, Ferro-Flores G. Radiopharmacokinetic and dosimetric parameters of 188Relanreotide in athymic mice with induced human cancer tumors. Int J Pharm. 2006; (310): 125-30.

22. Krylov V, Kochetova T, Smolyarchuk M, Petriev V, Kanygin V, Alexandrova A et al. Evaluation of 188Re-khedp (phosphoren) for palliative treatment in patients with bone metastases., World Journal of Nuclear Medicine. 2013; 12 (2): 255-6.

23. Kochetova T, Smolyarchuk M, Krylov V, Voloznev V, Lunev A, Petriev VM. 188Re-zoledronic acid: first in man study. World Journal of Nuclear Medicine. 2013; 12 (2): 228-9.

24. Konior M, Iller E. Classic Radionuclide 188W/188Re Generator (Experiments, Design and Construction). Mod Chem appl. 2014; (2): 143.

25. Evropejskaja konvencija po zashhite pozvonochnyh zhivotnyh, ispol'zuemyh dlja jeksperimental'nyh i drugih nauchnyh celej, EJeS, Strasburg, 1985 g. Lanimalogija. 1993; (1): 29 s.

26. Belenkiy ML. Jelementy kolichestvennoj ocenki farmakologicheskogo jeffekta: L.: Izd-vo «MedGiz», 1968; $151 \mathrm{~s}$.

27. Guskova TA. Toksikologiya lekarstvennyh sredstv: M.: Izd-vo "Russkij vrach», 2003; 154 s.

28. Trahtenberg IA, Sova AE, Sheftel VO, Onikienko FA. Problema normy v toksikologii. M.: Izd-vo «Medicina», 1992; $208 \mathrm{~s}$.

29. Seguin J, Nicolazzi C, Mignet N, Scherman D, Chabot GG. Vascular density and endothelial cell expression of integrin alpha $\checkmark$ beta 3 and E-selectin in murine tumours. Tumour Biol. 2012; 33 (5): 1709-17.

30. Treshhalina EM, Zhukova OS, Gerasimova GK, Andronova NV dr. Metodicheskie rekomendacii po doklinicheskomu izucheniju protivoopuholevoj aktivnosti lekarstvennyh sredstv. $\mathrm{V}$ knige: Mironov AN, Bunatjan ND i dr., redaktory. Rukovodstvo po provedeniju doklinicheskih issledovanij lekarstvennyh sredstv. M.: Grif i K., 2012; S. 642-657.

31. Koldaeva EYu, Grigorieva EYu, Stukalov YuV. Sravnitel'naja toksikologija nanokonstrukcij na osnove dendrimerov dlja targetnoj radioterapii $v$ onkologii. Rossijskij Bioterapevticheskij Zhurnal. 2010; 9 (3): 13-14.

32. Argyrou M, Valassi A, Andreou M, Lyra M. Rhenium-188 Production in Hospitals, by W-188/Re-188 Generator, for Easy Use in Radionuclide Therapy. Int J Mol Imaging. 2013; (2013): $1-7$. 